\title{
Recent developments in dynamic testing of materials
}

\author{
A. Gilat and J.D. Seidt \\ The Ohio State University, Department of Mechanical Engineering, Columbus OH, USA
}

\begin{abstract}
Three new testing configurations that have been developed since the last DYMAT conference in 2009 are presented. The first is high strain rate testing of Kevlar cloth and Kevlar yarn in a tensile Split Hopkinson Bar (SHB) apparatus. The Kevlar cloth/yarn is attached to the bars by specially designed adaptors that keep the impedance constant. In addition to determining the specimen's stress and strain from the recorded waves in the bars the deformations are also measured with Digital Image Correlation (DIC). The second testing configuration is a high strain rate shear test for sheet metal. The experiment is done by using a flat notched specimen in a tensile SHB apparatus. The shear strain is measured using DIC within the notch and on the boundary. The third development is a compression apparatus for testing at intermediate strain rates ranging from $20 \mathrm{~s}^{-1}$ to $200 \mathrm{~s}^{-1}$. The apparatus is a combination of a hydraulic actuator and a compression SHB. The stress in the specimen is determined from the stress wave in a very long transmitter bar and the strain and strain rate is determined by using DIC. The results show clean stress strain curves (no ringing).
\end{abstract}

\section{Background}

The Split Hopkinson Bar (SHB), introduced by Kolsky [1] in 1949 , is the most commonly used technique for characterizing the deformation and failure of materials at strain rates ranging from about $400 \mathrm{~s}^{-1}$ to $5,000 \mathrm{~s}^{-1}$. In this technique, a material specimen that is placed between two bars is loaded by a stress wave that is generated in one of the bars (incident bar). The force applied to the specimen and the average strain in the specimen can be determined from the waves in the bars that remain within the elastic limit during the experiment. The technique was introduced with compression loading and was later modified for tension and torsion loadings.

Over many years the SHB technique was mostly used for testing specimens that were subjected to a uniform state of stress and deformation. In these tests the force (or torque) in the specimen is determined from the wave in the transmitter bar and the average strain is calculated from the difference in the motion at the ends of the specimen's gage section that are determined from the waves in the bars. Recently, the Digital Image Correlation (DIC) method for strain measurement has been incorporated into the SHB test, Gilat et al [2]. DIC measures the full field deformation directly on the specimen and thus provides means for examining whether the deformation in the specimen is uniform and means for conducting tests where in deformation between the ends of the bars is intentionally not uniform.

Three dynamic tests associated to the SHB technique with DIC are presented in this paper. In the first application, Kevlar yarn and cloth is tested in a tensile SHB apparatus. Kevlar is sometimes used in applications where dynamic loadings are applied, and the tests were done in order to check whether the mechanical properties of Kevlar are sensitive to the strain rate. The second test is a high strain rate shear of sheet metal. The test is done by using a flat notched specimen in a tensile SHB. Dynamic shear tests are usually done with a torsional SHB using spoolshaped specimens which cannot be machined from sheet metal.
The third experiments deals with testing materials at intermediate strain rates between $20 \mathrm{~s}^{-1}$ and $200 \mathrm{~s}^{-1}$. Testing at this range is difficult because the strain rates are too low for the standard split Hopkinson bar technique and are too high for standard hydraulic machines. Deforming a specimen to an appreciable strain takes milliseconds and in a typical SHB apparatus the loading lasts well less than one millisecond. On the other end the actuator of a typical hydraulic machine can move fast enough to deform the specimen at the required strain rate, but results from such tests are noisy with large oscillations (referred to as ringing). This happens because the whole testing machine is not in static equilibrium during the test. To overcome these difficulties some researchers have modified the standard hydraulic machine and/or the method by which the data is analyzed, and others have modified the SHB technique. Song et al. [3] have used a compression SHB with a long bars for testing soft materials. Zhao and Gary [4], and Othman and Gary [5] have used a hydraulic actuator for long loading pulse and separation of waves technique for analyzing the data. The new apparatus for compression testing at intermediate strain rates that is presented here is made up from a hydraulic actuator that can apply dynamic loads for the required duration and a long transmitter bar that can measure the force in the specimen without the effects of wave reflections.

\section{Tensile testing of Kevlar in SHB}

Testing Kevlar cloth and yarn requires special attention to the mounting, or gripping, of the specimen. At low (quasistatic) strain rates there are no limits on the specimen and mounting fixtures geometries. Testing these materials with the tensile SHB apparatus requires special attention since the specimen has to be small, should be attached between the bars while keeping the impedance constant, and the connection has to be strong enough such that the specimen will fracture in the gage section between the bars. The tensile SHB in our lab is made of Aluminum 


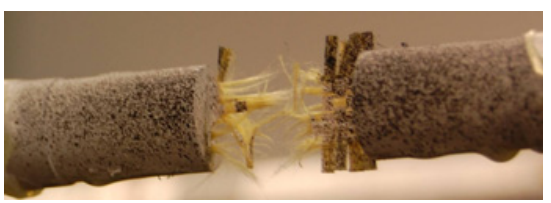

Fig. 1. Kevlar cloth specimen following a tensile SHB test.

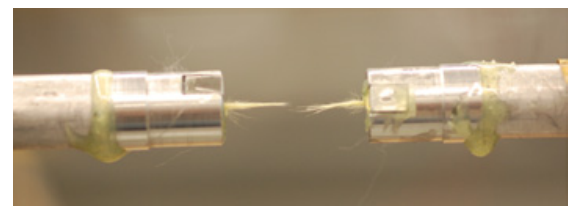

Fig. 2. Kevlar yarn specimen following a tensile SHB test.

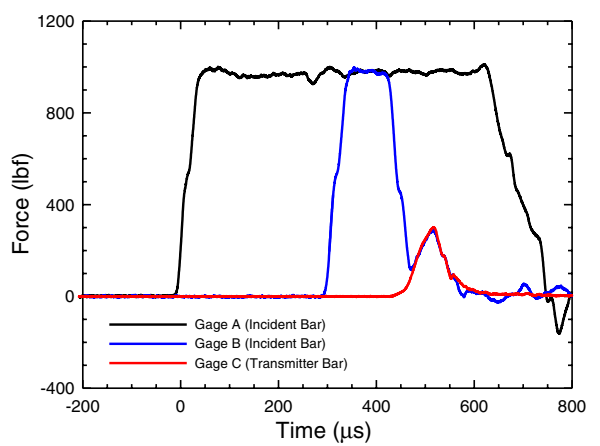

Fig. 3. Waves recorded in a tensile SHB test on Kevlar cloth.

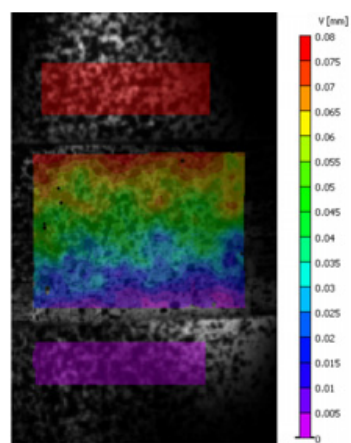

Fig. 4. DIC data from a tensile SHB test on Kevlar cloth.

(7075-T651) with a diameter of $12.7 \mathrm{~mm}$. Several fixtures for attaching Kevlar specimens have been tried. Figures 1 and 2 show fractured Kevlar 49 cloth specimen and fractured single yarn specimen following a test.

The stress and strain in the specimen are determined from the wave in the bar using the standard method. In addition, the strain is determined with DIC directly on the specimen and from measuring the displacements of the adaptors. Figure 3 shows the waves recorded during a SHB test and figure 4 shows DIC data (displacements) from one frame. Figure 5 shows processed data in the form of stress and strain rate plots versus time and figure 6 shows stress strain curves from a test with Kevlar 49 cloth.

\section{High strain rate shear testing of sheet metal}

A new tensile SHB configuration for shear testing of sheet metal specimens is presented. In this configuration a flat

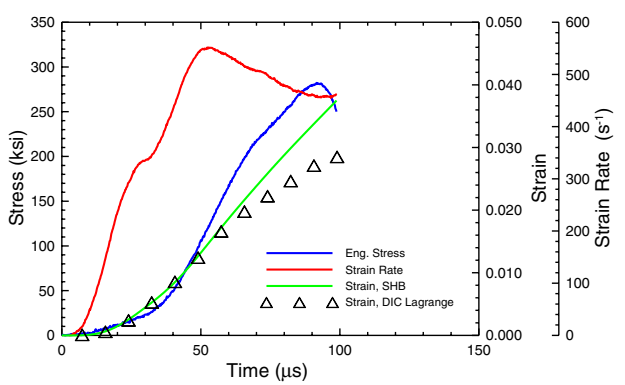

Fig. 5. Stress, strain, and strain rate during a tensile SHB test on Kevlar cloth.

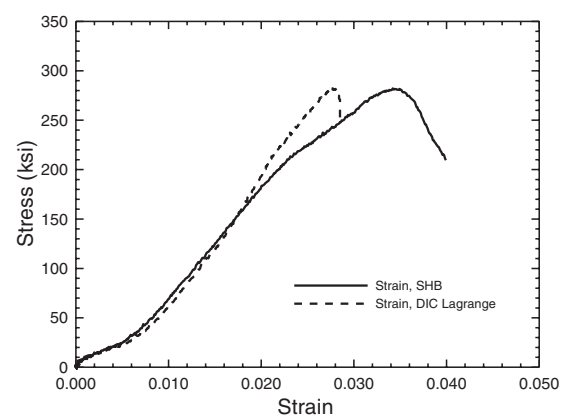

Fig. 6. Stress strain curve from a tensile SHB test on Kevlar cloth.

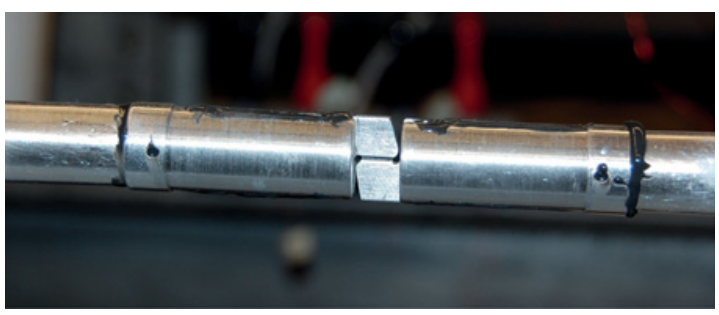

Fig. 7. Notched specimen for shear testing in tensile SHB.
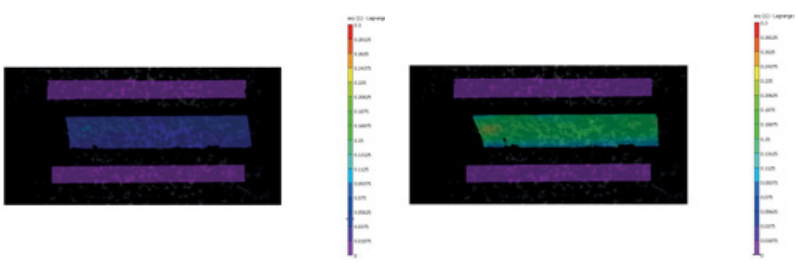

Fig. 8. Shear strain in the gage section measured with DIC.

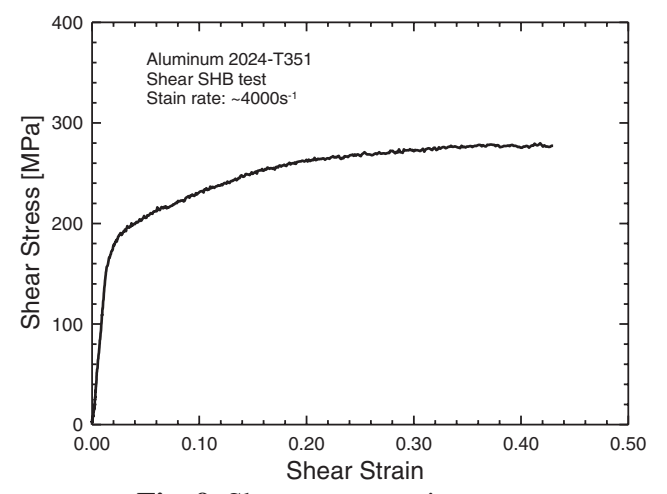

Fig. 9. Shear stress strain curve.

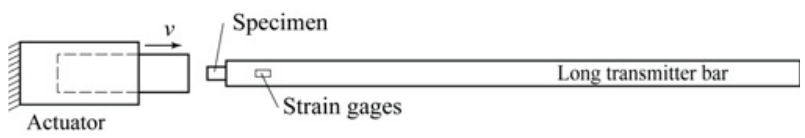

Fig. 10. Schematic of the intermediate strain rate apparatus. 


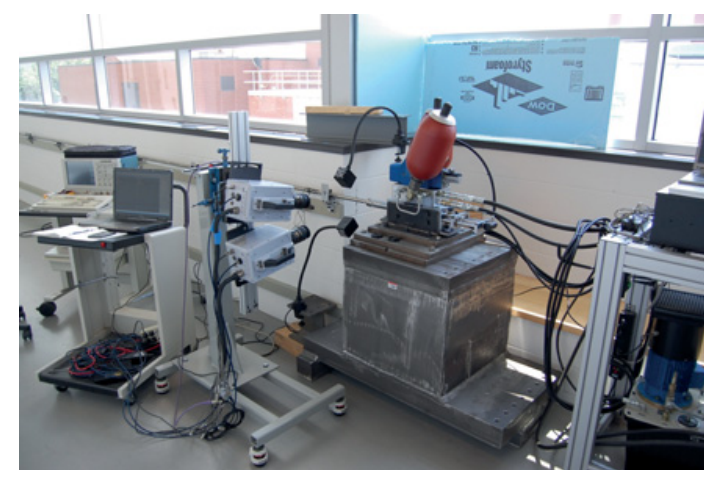

Fig. 11. Intermediate strain rate apparatus.

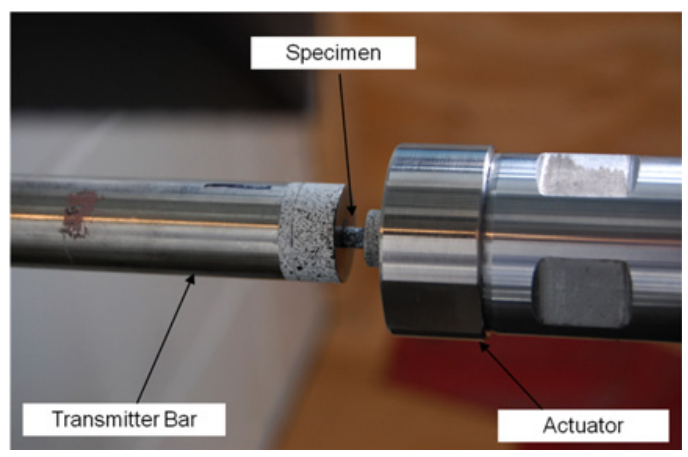

Fig. 12. Intermediate strain rate apparatus.

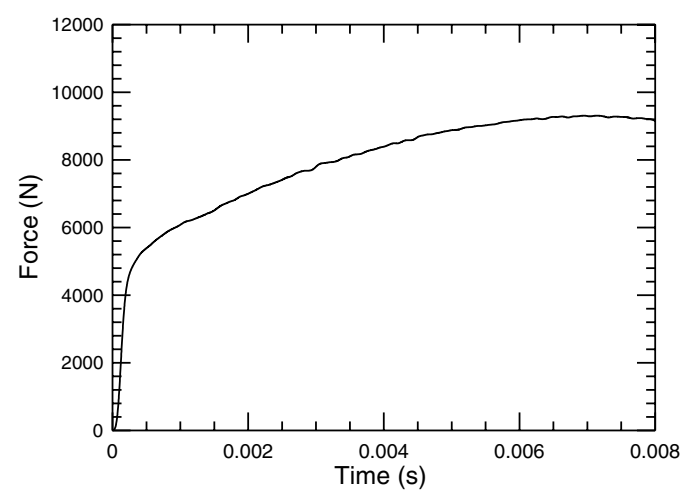

Fig. 13. Force record in a test with 6061-T6 aluminium.

notched specimen is glued to the bars using adaptors, Figure 7. The average shear stress in the gage section is determined from the force measured in the transmitter bar. The shear strain is determined by measuring the deformation with DIC directly in the gage section.

DIC results from two frames during a test are shown in figure 8. The images show a state of nearly pure and uniform shear strain within the gage section. A shear stress stain curve obtained in a test at strain rate of about $4000 \mathrm{~s}^{-1}$ is shown in figure 9.

\section{Intermediate strain rate test}

A new compression testing technique at intermediate strain rates ranging from about $20 \mathrm{~s}^{-1}$ to $200 \mathrm{~s}^{-1}$ is presented. The technique is a hybrid of a compression SHB and

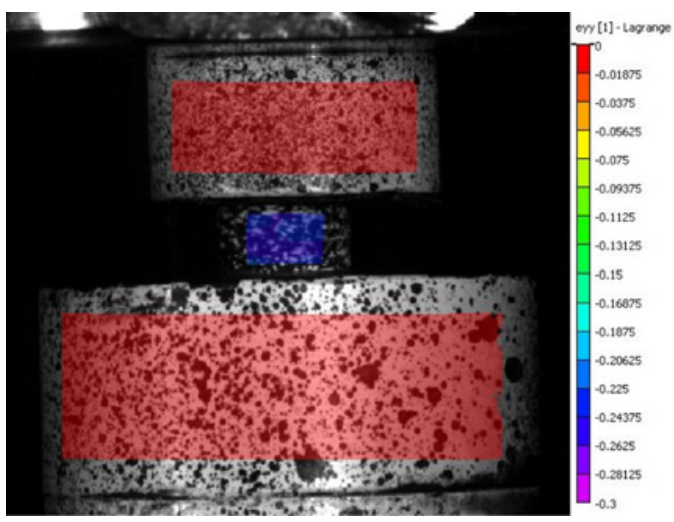

Fig. 14. Axial strain measured with DIC in a compression test.

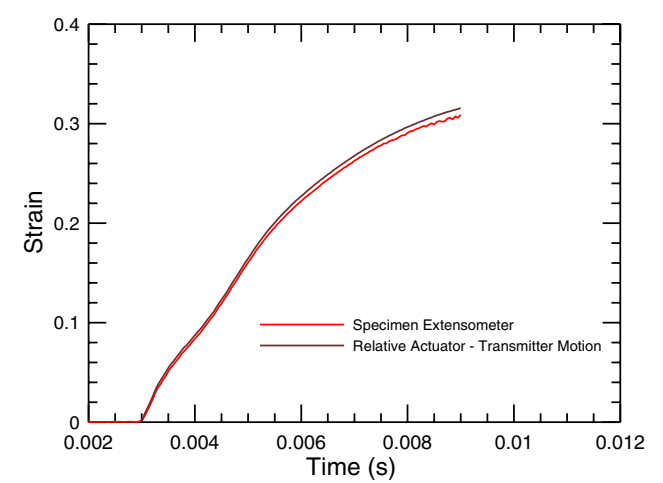

Fig. 15. Axial engineering strain vs. time determined by DIC.

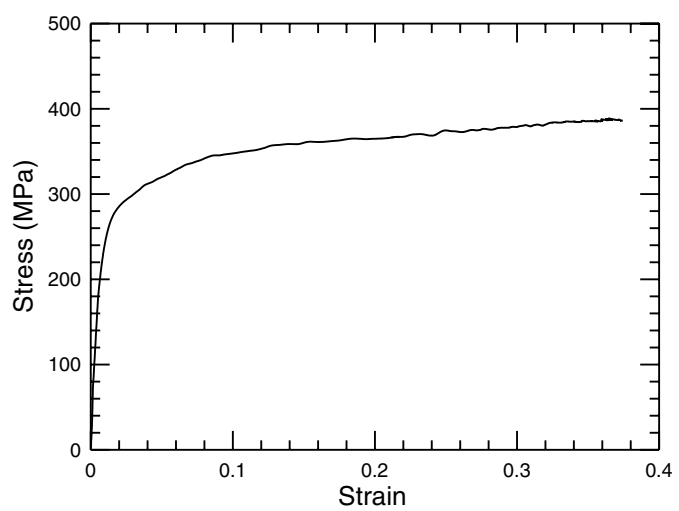

Fig. 16. Stress strain curve for 6061-T6 aluminium.

a hydraulic machine. A specimen (short, small diameter cylinder) that is placed on the face of the cross-section of a long bar is loaded by a hydraulic actuator, figure 10. As the specimen is loaded, a compression wave propagates to the end of the bar and reflects back. The force in the specimen is measured by strain gages that are placed on the bar and the strain in measured directly on the specimen with DIC. The actual setup is shown in figures 11 and 12 .

The bar is more than $40 \mathrm{~m}$ long which allows a test duration (until the reflected wave arrives at the strain gages that measure the force) of more than $0.016 \mathrm{~s}$. At a strain rate of $20 \mathrm{~s}^{-1}$ it provides enough time for the specimen to deform to a strain of 0.3. Results from a test on a 6061-T6 aluminium specimen are shown in figures 13-16. Figure 13 shows the force recorded in the specimen during a test 
and figure 14 shows one DIC frame during the test. The engineering axial strain determined by DIC is shown in figure 15 . The strain rate determined from the slope of the curve is approximately $70 \mathrm{~s}^{-1}$. The stress strain curve from the test is shown in figure 16.

\section{Acknowledgements}

The research on the properties of Kevlar was supported by the U.S.A. Federal Aviation Administration, Grant No. 2006G004. The shear test with sheet metal was initially developed by Mr. Matti Isakov from Tampere University of Technology, Finland. Additional tests and modifications were done by Mr. Kevin Gardner. The development of the intermediate strain rate test was done by Mr. Thomas Matrka with support from NASA, Grant No. NNX08AB50A.

\section{References}

1. H. Kolsky, Proc Phys Soc London, B62, 676 (1949)

2. A. Gilat, T.E. Schmidt, A.L. Walker, Exp. Mech., 49, 291, (2009)

3. B. Song, C.J. Syn, C.L. Grupido, W. Chen, and W.-Y. Lu, Exp. Mech., 48, 809 (2008)

4. H. Zhao and G. Gary, J. Mech. Phys. Solids, 45, 1185 (1997).

5. R. Othman, and G. Gary, Exp. Mech. 47, 295 (2007) 